\title{
Reduction of U(VI) and Toxic Metals by Desulfovibrio Cytochrome c3
}

\author{
Wall, Judy D. \\ University of Missouri
}

\begin{abstract}
RESULTS TO DATE: The project, "Reduction of U(VI) and toxic metals by Desulfovibrio cytochrome c3," is designed to obtain spectroscopic information for or against a functional interaction of cytochrome c3 and uranium in the whole cells. That is, is the cytochrome c3 the uranium reductase? Our approach has been to start with purified cytochrome and determine any unique spectral disturbances during electron flow to $\mathrm{U}(\mathrm{VI})$. Then we will attempt to identify these signals emanating from cells actively reducing uranium. This project is being carried out in collaboration with Dr. William Woodruff at the Los Alamos National Laboratory where the spectral experiments are being carried out.
\end{abstract}

We at the University of Missouri are preparing purified cytochrome $\mathrm{c} 3$ for the experiments. We have a procedure that appears to give $>90-95 \%$ purity of this cytochrome from periplasmic extracts of Desulfovibrio desulfuricans strain G20. Our yields are in the range of 0.14-0.16 mg/l cell culture. These small batches of pure protein are being used for the baseline experiments.

We have contacted the Protein Core Facility of the University of Missouri to set up crystallization trials for this cytochrome. Crystal structures of several c-type cytochromes have been published. Therefore, we anticipate reasonable progress in obtaining the structure of this protein from strain G20. From this information a more informed approach to site-directed mutagenesis of important residues will be possible. In addition, the crystallization of candidate mutants can be compared to that of the wild-type protein to determine subtle structural alterations resulting from the mutations.

Production of the cytochrome and any mutant proteins generated in the future can be made more efficient by cloning of the genes into a protein expression vector that may be used either in Escherichia coli or Desulfovibrio. We have obtained a candidate expression vector from IBA GmbH, Gottingen, Germany, and are attempting to subclone the wild-type gene. Several features of the expression vector will need to be tested in the sulfate-reducing bacteria to allow it to be usefully applied in these bacteria. A replicon conferring stability in the sulfate reducers will be added to the vector as well.

A deletion of the wild-type gene for cytochrome $\mathrm{c} 3$ is being constructed so that recombinant cytochrome c3 can be produced without contamination of the wild-type protein. A mutagenic construct has been made with $0.9 \mathrm{~kb}$ upstream and $1.1 \mathrm{~kb}$ downstream of the gene flanking a kanamycin resistance cassette. This construct has been introduced into the strain G20 and kanamycin resistance selected. The single recombination events that have inserted the plasmid are now being characterized before proceeding to the screen for the second event that will delete the gene and the plasmid from the cells.

DELIVERABLES: No papers have resulted from this project to date. 